\title{
Asymmetry Superhydrophobic Textiles for Electromagnetic Interference Shielding, Photothermal Conversion, and Solar Water Evaporation
}

En Li ${ }^{\dagger}, \#$, Yamin Pan ${ }^{\dagger, \#}$, Chunfeng Wang ${ }^{\ddagger}$, Chuntai Liu ${ }^{\dagger}$, Changyu Shen ${ }^{\dagger}$, Caofeng Pan ${ }^{\ddagger}$, Xianhu Liu $^{* \dagger}$

$\dagger$ College of Materials Science and Engineering, National Engineering Research Center for Advanced Polymer Processing Technology, Henan Province Industrial Technology Research Institute of Resources and Materials, Key Laboratory of Advanced Material Processing \& Mold (Ministry of Education), Zhengzhou University, Zhengzhou, 450002, China

* Beijing Institute of Nanoenergy and Nanosystems, Chinese Academy of Sciences, National Center for Nanoscience and Technology (NCNST), Beijing, 100083, China

*Corresponding author.

Email:xianhu.liu@zzu.edu.cn

\# These authors contributed equally to this work 
1. Supporting Figures

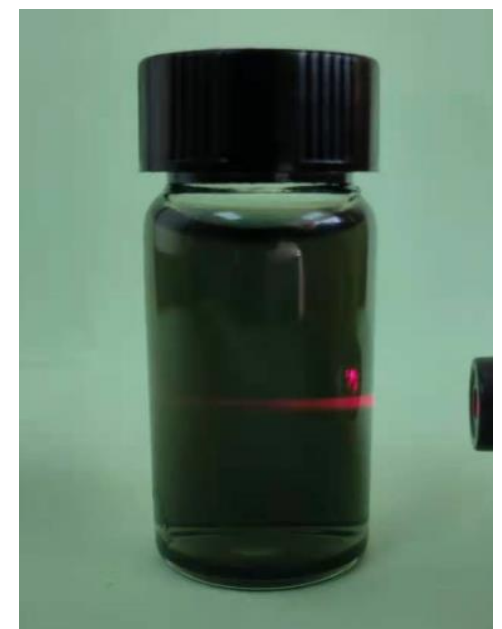

Figure S1. Tyndall effect of MXene solution. 


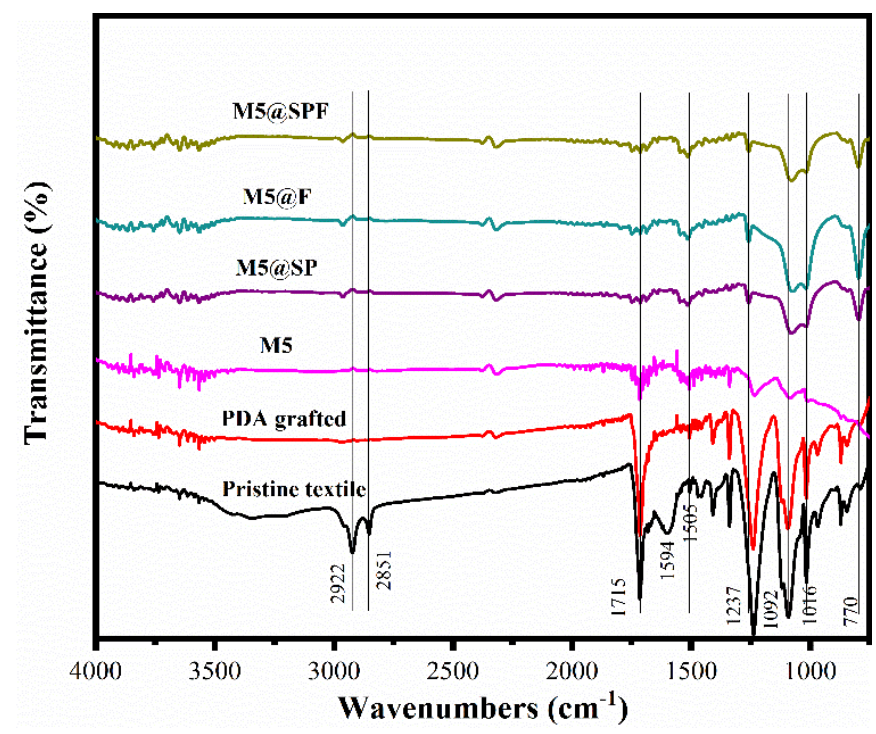

Figure S2. FTIR spectra of pristine textile, PDA grafted textile, M5 textile, M5@SP textile, M5@F textile,M5@SPF textile. 


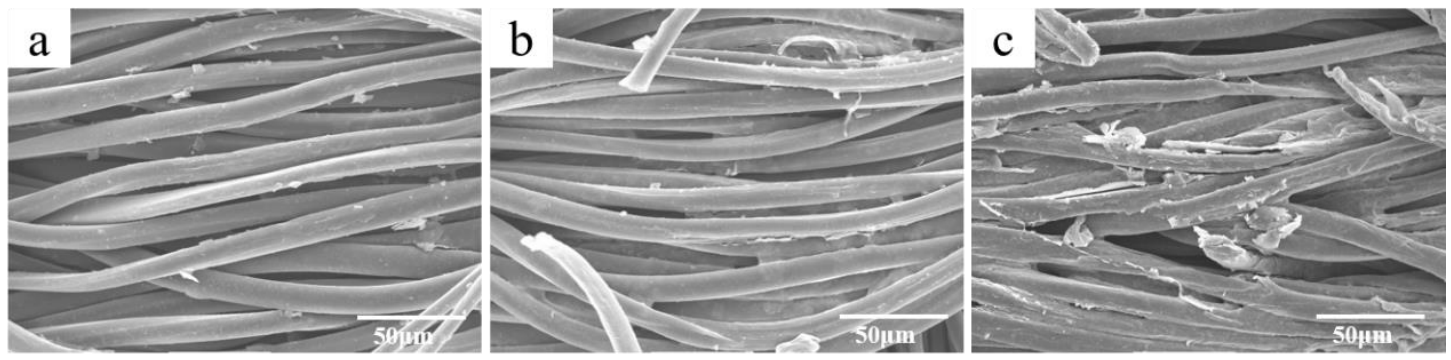

Figure S3. SEM images of (a) M1 textile, (b) M3 textile, (c) M7 textile. 


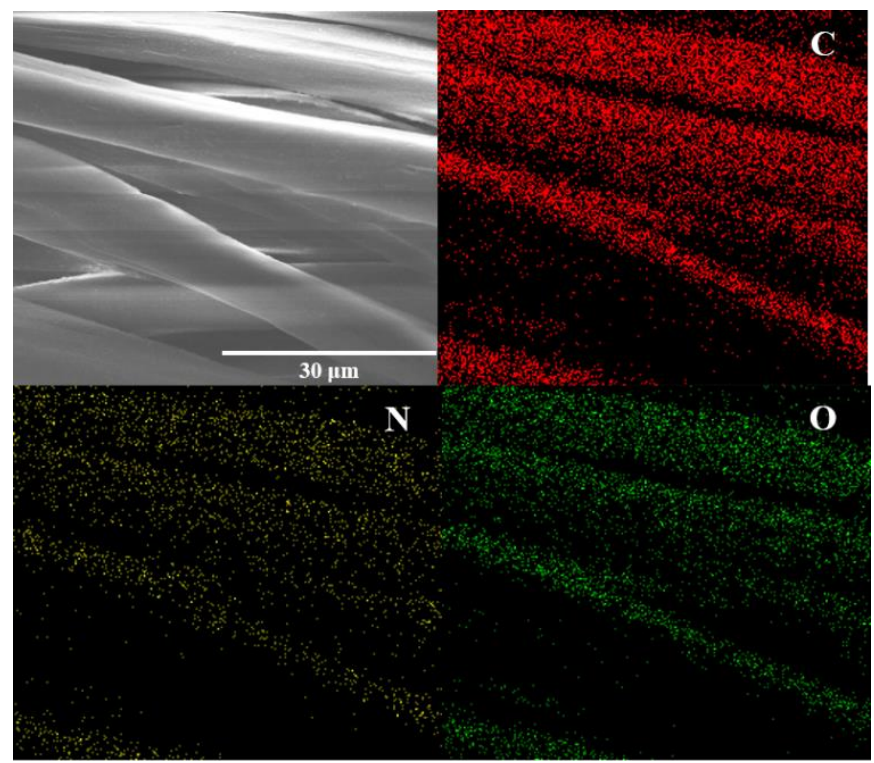

Figure S4. EDS elemental mapping images of PDA grafted textile. 


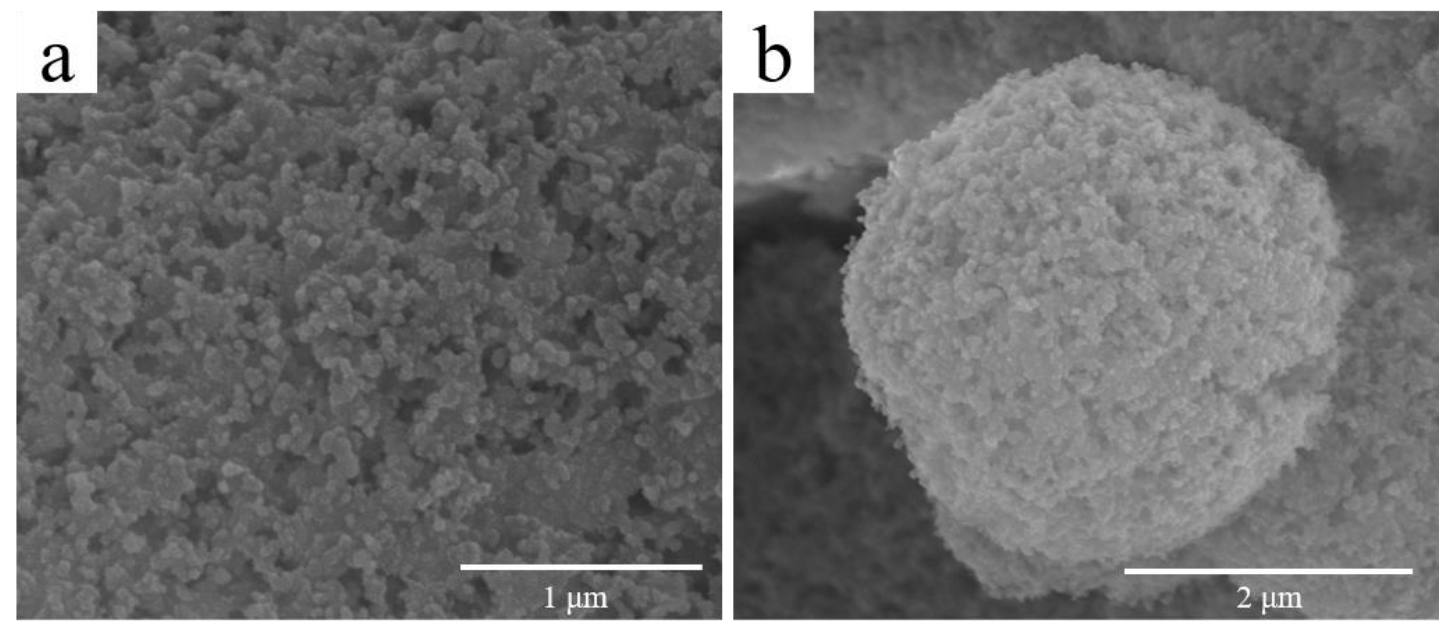

Figure S5. SEM images of (a) $\mathrm{SiO}_{2} / \mathrm{PDMS}$ coating, (b) $\mathrm{SiO}_{2} / \mathrm{PDMS}$ agglomerated microspheres. 


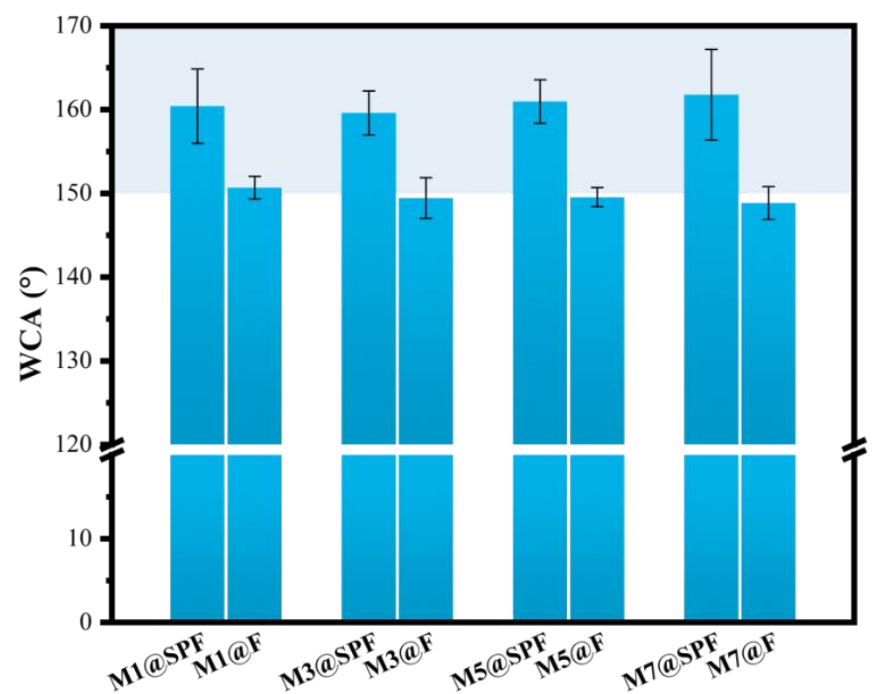

Figure S6. WCAs of the Mx@SPF and Mx@F textiles. 


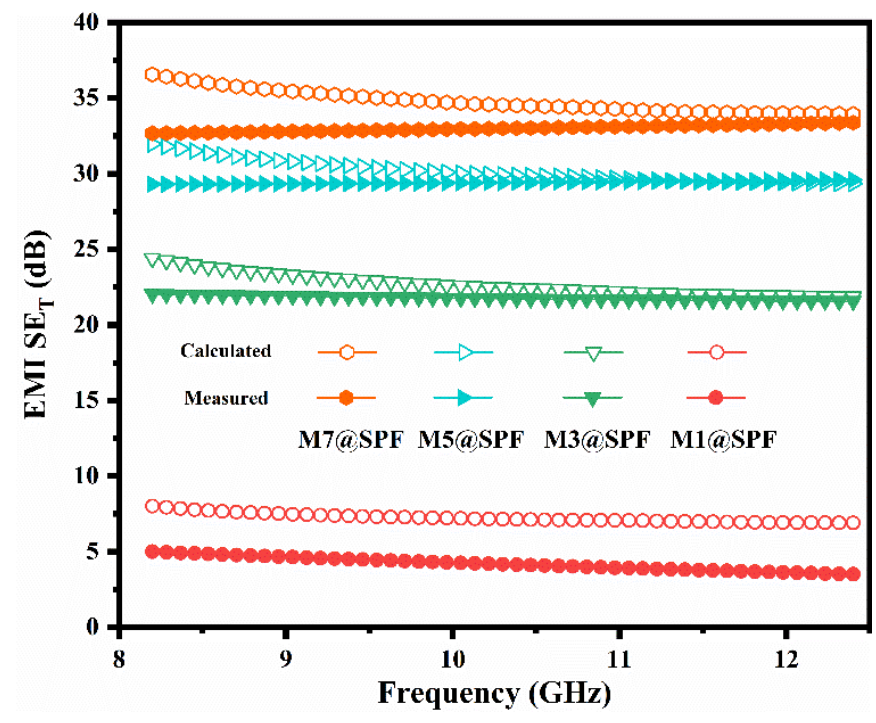

Figure S7. The comparison between the theoretical $\mathrm{SE}_{\mathrm{T}}$ value of EMI calculated by Simon's formula and the experimental result. 


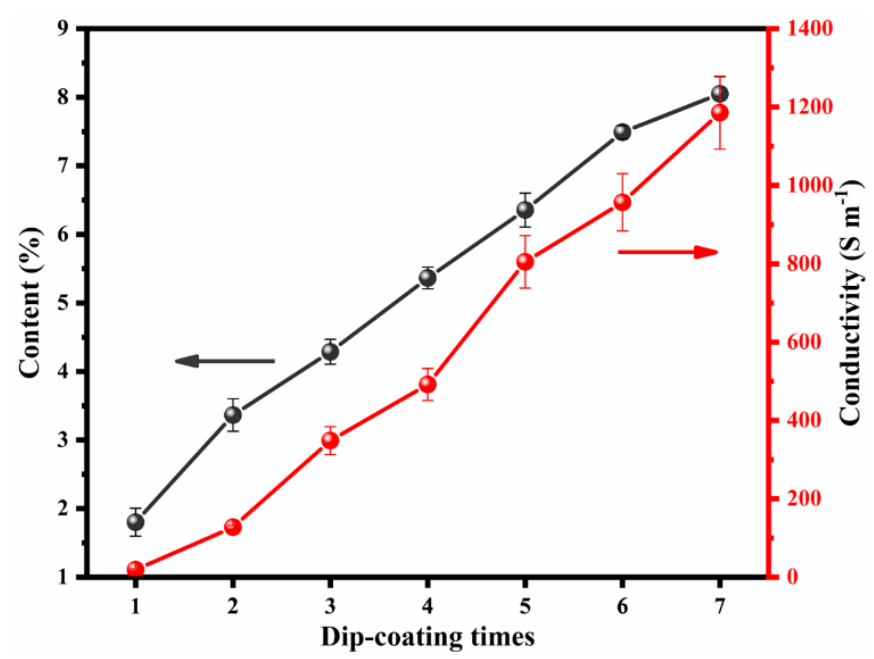

Figure S8. Electrical conductivity and MXene content of Mx textile with different dip-coating times. 

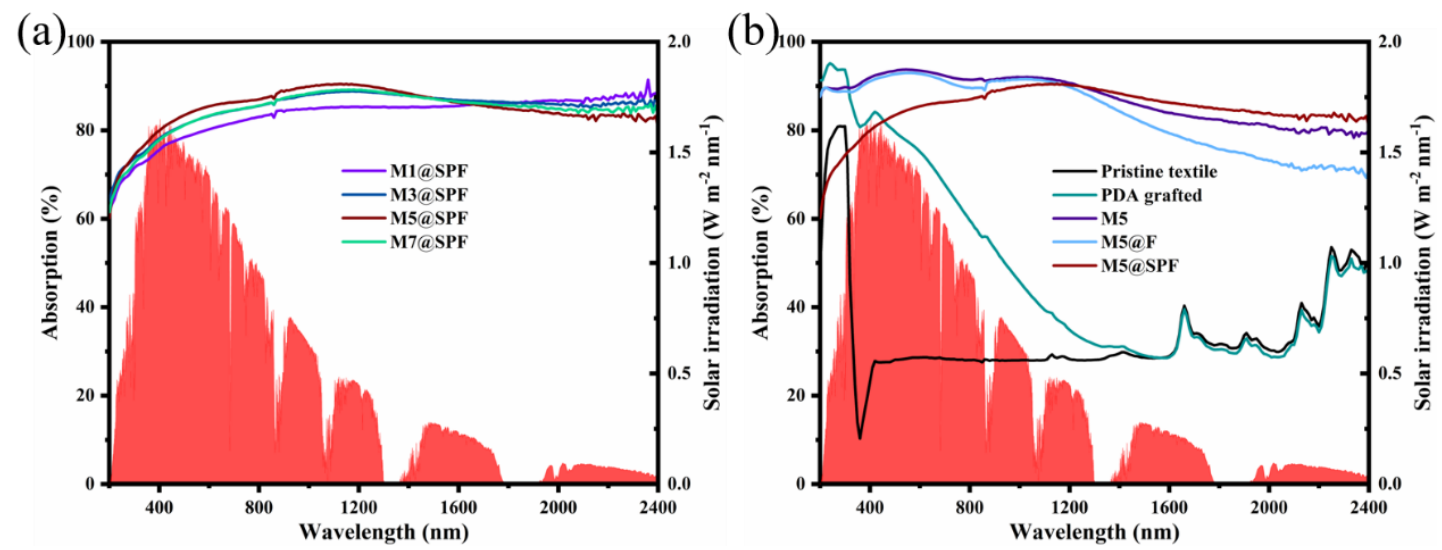

Figure S9. Absorption spectra of (a) Mx@SPF textiles, and (b) pristine textile, PDA grafted textile, M5 textile, M5@F textile, M5@SPF textile, and the solar spectral irradiation weighted by standard AM 1.5G solar spectrum. 


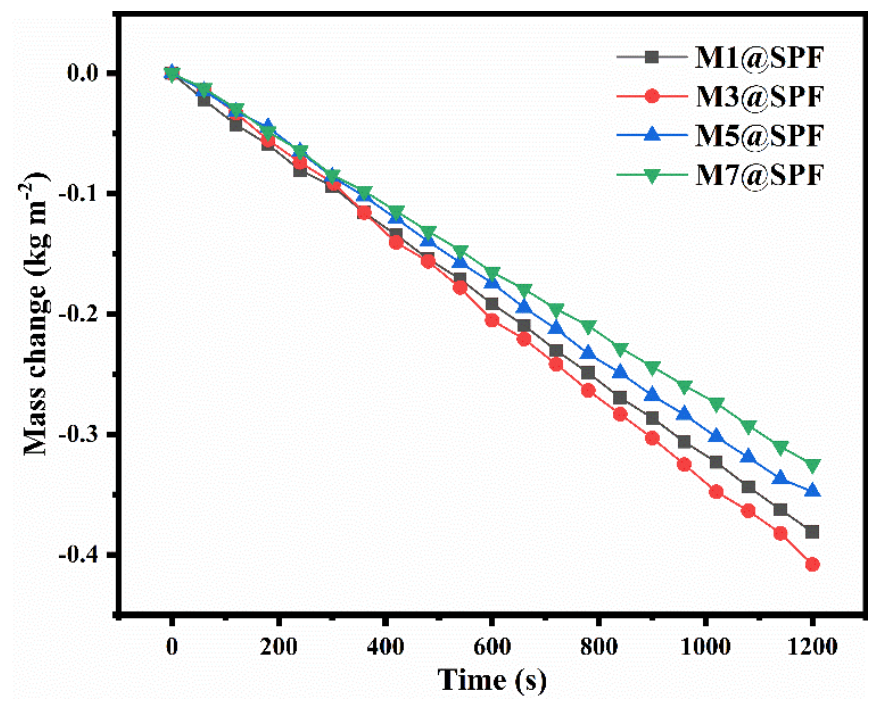

Figure S10. Mass change of water overtime for the Mx@SPF textiles with reservoir under the power density of $100 \mathrm{~mW} / \mathrm{cm}^{2}$. 
2. Supporting Table

Table S1. Comparison of EMI SE of various EMI shielding textiles.

\begin{tabular}{|c|c|c|c|c|c|}
\hline Materials & Thickness & Conductivity & $\begin{array}{c}\text { Test } \\
\text { range }\end{array}$ & $\begin{array}{l}\text { EMI SE } \\
\text { (dB) }\end{array}$ & Ref \\
\hline $\begin{array}{l}\text { MXene/PPy } \\
\text { PET textile }\end{array}$ & $0.45 \mathrm{~mm}$ & $1000 \mathrm{~S} / \mathrm{m}$ & $\begin{array}{c}\text { 8.2-12.4 } \\
\mathrm{GHz}\end{array}$ & 42 & 1 \\
\hline $\begin{array}{c}\text { AgNPs/Fe3O4/PDMS } \\
\text { PP fabric }\end{array}$ & NA & $108.8 \mathrm{~S} / \mathrm{cm}$ & $\begin{array}{c}\text { 8.2-12.4 } \\
\text { GHz }\end{array}$ & 56.1 & 2 \\
\hline $\begin{array}{c}\text { MXene } \\
\text { PET textile }\end{array}$ & $0.34 \mathrm{~mm}$ & $117 \mathrm{~S} / \mathrm{m}$ & $\begin{array}{c}\text { 8.2-12.4 } \\
\text { GHz }\end{array}$ & 42.1 & 3 \\
\hline $\begin{array}{c}\text { MXene } \\
\text { fabric grid }\end{array}$ & $0.5 \mathrm{~mm}$ & NA & $\begin{array}{c}\text { 8.2-12.4 } \\
\text { GHz }\end{array}$ & 38.8 & 4 \\
\hline $\begin{array}{l}\text { MXene/AgNWs } \\
\text { silk textile }\end{array}$ & $0.12 \mathrm{~mm}$ & $0.8 \Omega$ sq-1 & $\begin{array}{c}\text { 8.2-12.4 } \\
\text { GHz }\end{array}$ & 54 & 5 \\
\hline $\begin{array}{l}\text { Ni-W-P } \\
\text { PA fabric } \\
\end{array}$ & $0.117 \mathrm{~mm}$ & NA & $\begin{array}{c}2-12.5 \\
\text { GHz }\end{array}$ & 43.6 & 6 \\
\hline $\begin{array}{c}\text { CNT/Graphene/WPU } \\
\text { textile }\end{array}$ & $0.35 \mathrm{~mm}$ & NA & $\begin{array}{c}\text { 8.2-12.4 } \\
\text { GHz }\end{array}$ & 35 & 7 \\
\hline $\begin{array}{l}\text { PEDOT/MXene } \\
\text { cotton fabric }\end{array}$ & $\mathbf{N A}$ & $3.6 \Omega \mathrm{sq}-1$ & $\begin{array}{c}\text { 8.2-12.4 } \\
\mathrm{GHz}\end{array}$ & 36.62 & 8 \\
\hline $\begin{array}{c}\text { MXene/SiO2/PDMS/PFOTES } \\
\text { PET textile }\end{array}$ & $0.2 \mathrm{~mm}$ & $200 \mathrm{~S} / \mathrm{m}$ & $\begin{array}{c}\text { 8.2-12.4 } \\
\text { GHz }\end{array}$ & 36.6 & $\begin{array}{l}\text { This } \\
\text { work }\end{array}$ \\
\hline
\end{tabular}

Notes: PPy: polypyrrole; PET: Polyethylene terephthalate; AgNPs: silver nanoparticles; PDMS: polydimethylsiloxane; PP: polypropylene; AgNWs: silver nanowires; Ni: nickel; W: tungsten; P: phosphorus; PA: polyamide; CNT: carbon nanotube; WPU: waterborne polyurethane; PEDOT: poly(3,4-ethylenedioxythiophene). 


\section{Supporting Movies}

Movie S1: The water repellency of the SPF surface of the superhydrophobic textile under the impact of water flow.

Movie S2 and Movie S3: M5@SPF textile has superhydrophobic properties while maintaining good air permeability.

Movie S4: Self-cleaning performance of the surface of M5@F and M5@SPF textiles.

Movie S5: The mass change of water overtime for the M3@SPF textile with reservoir. 


\section{References}

1. Wang, Q.; Zhang, H.; Liu, J.; Zhao, S.; Xie, X.; Liu, L.; Yang, R.; Koratkar, N.; Yu, Z. Multifunctional and Water-Resistant MXene-Decorated Polyester Textiles with Outstanding Electromagnetic Interference Shielding and Joule Heating Performances. Adv. Funct. Mater. 2019, 29 (7), 1806819, DOI: $\underline{\text { https://doi.org/10.1002/adfm.201806819. }}$.

2. Luo, J.; Huo, L.; Wang, L.; Huang, X.; Li, J.; Guo, Z.; Gao, Q.; Hu, M.; Xue, H.; Gao, J. Superhydrophobic and Multi-responsive Fabric Composite with Excellent Electro-photo-thermal Effect and Electromagnetic Interference Shielding Performance. Chem. Eng. J. 2020, 391, 123537, DOI: $10.1016 /$ j.cej.2019.123537.

3. Liu, X.; Jin, X.; Li, L.; Wang, J.; Yang, Y.; Cao, Y.; Wang, W. Air-permeable, Multifunctional, Dual-Energy-driven MXene-decorated Polymeric Textile-based Wearable Heaters with Exceptional Electrothermal and Photothermal Conversion Performance. J. Mater. Chem. A 2020, 8 (25), 12526-12537, DOI: 10.1039/d0ta03048a.

4. Jia, X.; Shen, B.; Zhang, L.; Zheng, W. Waterproof MXene-decorated Wood-pulp Fabrics for High-Efficiency Electromagnetic Interference Shielding and Joule Heating. Compos. Pt. B-Eng. 2020, 198, 108250, DOI: 10.1016/j.compositesb.2020.108250.

5. Liu, L. X.; Chen, W.; Zhang, H. B.; Wang, Q. W.; Guan, F.; Yu, Z. Z. Flexible and Multifunctional Silk Textiles with Biomimetic Leaf-Like MXene/Silver Nanowire Nanostructures for Electromagnetic Interference Shielding, Humidity Monitoring, and Self-Derived Hydrophobicity. Adv. Funct. Mater. 2019, 29 (44), 1905197, DOI: 10.1002/adfm.201905197.

6. Bai, Y.; Qin, F.; Lu, Y. Multifunctional Electromagnetic Interference Shielding Ternary Alloy (Ni-W-P) Decorated Fabric with Wide-Operating-Range Joule Heating Performances. ACS Appl. 
Mater. Interfaces 2020, 12 (42), 48016-48026, DOI: 10.1021/acsami.0c15134.

7. Dai, M.; Zhai, Y.; Zhang, Y. A Green Approach to Preparing Hydrophobic, Electrically Conductive Textiles Based on Waterborne Polyurethane for Electromagnetic Interference Shielding with Low Reflectivity. Chem. Eng. J. 2020, 127749, DOI: 10.1016/j.cej.2020.127749.

8. Zheng, X.; Shen, J.; Hu, Q.; Nie, W.; Wang, Z.; Zou, L.; Li, C. Vapor Phase Polymerized Conducting Polymer/MXene Textiles for Wearable Electronics. Nanoscale 2021, 13 (3), 1832-1841, DOI: $10.1039 / \mathrm{d} 0 \mathrm{nr} 07433 \mathrm{k}$. 